Bruce ALBERT, François-Michel LE TOURNEAU:

\title{
« Homoxi : ruée vers l’or chez les Indiens Yanomami du haut rio Mucajaí (Brésil) »
}

Société amérindienne de chasseurs-cueilleurs et horticulteurs pratiquant la culture itinérante sur brûlis, les Yanomami occupent un territoire d'environ 192000 km² situé entre le Brésil et le Venezuela, de part et d'autre de la chaîne de la Serra Parima. Des années 1910 aux années 1960 les Yanomami du Brésil n’ont connu que des contacts sporadiques avec le monde « blanc ». Ils connaîtront pour la première fois, entre 1973 et 1976, une forme de contact plus intense, durant la construction d'un tronçon de la route Perimetral Norte dans le sud-est de leur territoire. Après une décennie de répit relatif, le projet Calha Norte (1985-86) et, surtout, la ruée vers l'or du Roraima (1987-89), intensifieront brutalement l'avancée de la société régionale sur le territoire des ces Indiens, déclenchant de très vives réactions de protestation tant au Brésil que dans le monde et aboutissant, en 1992, à l'homologation de leurs terres sous forme d'une aire protégée de 96650 km², la Terra Indígena Yanomami. Dans les années suivantes, une structure d'assistance sanitaire, d'abord précaire puis de plus en plus efficace, a rendu possible une reprise de l'essor démographique de cette ethnie. Malgré ces succès, dans les régions où l'impact des activités minières clandestines a été le plus intense, les groupes locaux yanomami ont connu, depuis la fin des années 1980, des changements sociaux et économiques importants. Durement affectés sur le plan démographique et environnemental, ils ont opéré une série de réaménagements de leur mode traditionnel d'occupation de l'espace forestier en fonction de la présence des chercheurs d'or puis de la structure d'assistance installée durant le processus de leur expulsion. Cet article propose l'analyse d'un exemple de cette géométrie variable des stratégies spatiales yanomami face au contact, ceci à partir d'une étude détaillée de la région du haut rio Mucajaí dite "Homoxi ». A cette fin, nous décrivons dans un premier temps le contexte économique et géopolitique régional, en particulier la ruée vers l'or dans l'état de Roraima à la fin des années 1980, peu documentée dans la littérature scientifique. Changeant d'échelle, nous étudions ensuite l'histoire de trois communautés de la région de Homoxi (Tirei, Xere u et Yaritha, totalisant, à l'époque de l'enquête de terrain, quelque 360 personnes) et le processus de transformation de leur modèle spatial et productif, d'abord face l'invasion des orpailleurs, puis, après l'éviction de ces derniers, face à la structure d'assistance locale mise en place (FNS, FUNAI). Nous mettont ainsi en évidence la flexibilité du système social yanomami et sa capacité à générer des adaptations rapides aux situations les plus adverses.

Mots clés : Anthopologie, Géographie, Amazonie, Brésil, Roraima, amérindiens, Yanomami, changement social, usage de l'espace, chercheurs d'or, politique indigéniste 


\title{
Homoxi : ruée vers l’or chez les Indiens Yanomami du haut rio Mucajaí (Brésil)
}

\author{
Bruce Albert* \\ et \\ François-Michel Le Tourneau **
}

Société amérindienne de chasseurs-cueilleurs et horticulteurs pratiquant la culture itinérante sur brûlis, les Yanomami occupent un territoire d'environ $192000 \mathrm{~km}^{2}$ situé entre le Brésil et le Venezuela, de part et d'autre de la chaîne de la Serra Parima. Leur population globale est estimée à près de 29000 personnes, dont environ 14000 - soit 250 communautés vivant au Brésil ${ }^{1}$ dans la région du haut rio Branco (ouest de l'état de Roraima) et sur la rive gauche du rio Negro (nord de l'état d'Amazonas). Au terme d'une longue succession de projets et contre-projets officiels (1977-1991), le territoire des Yanomami du Brésil a été définitivement homologué en mai 1992 au titre de " terre indigène », catégorie d'aire protégée du domaine public réservant aux amérindiens « l'usufruit exclusif » de leurs territoires et des ressources naturelles qu'ils contiennent ${ }^{2}$. Les $96650 \mathrm{~km}^{2}$ (Figure 1) de cette vaste zone abritent une grande diversité de milieux naturels et sont considérés par la communauté scientifique comme une région prioritaire en matière de protection de la biodiversité amazonienne (voir Capobianco éd. 2001: 398-399).

Des années 1910 aux années 1960 les Yanomami du Brésil n’ont connu que des contacts sporadiques et limités avec des collecteurs de produits forestiers, quelques explorateurs étrangers ${ }^{3}$, des militaires de la Commission des frontières (CBDL), des agents du Service de Protection des Indiens (SPI) puis des missionnaires. ${ }^{4}$ Ils connaîtront pour la première fois, entre 1973 et 1976, une forme de contact plus intense, durant la construction d'un tronçon de la route Perimetral Norte dans le sud-est de leur territoire ${ }^{5}$. Après une décennie de répit relatif, le projet Calha Norte $(1985-86)^{6}$ et, surtout, la ruée vers l'or du Roraima (1987-89), intensifient brutalement l'avancée de la société régionale sur le territoire yanomami (Albert 1987, 1990 et 1992), aboutissant à deux résultats opposés.

D’un côté, l'invasion massive des terres indiennes par les chercheurs d'or a menacé de provoquer la disparition de l'ethnie, notamment du fait des constantes épidémies de paludisme et de grippe, consécutives au contact, et de l'absence de toute assistance médicale durant cette période. D’un autre côté, la pression internationale exercée sur le Brésil à propos du "génocide des Yanomami » a permis de débloquer le dossier de la reconnaissance officielle d'un territoire protégé, dont les premières revendications avaient émergé dès la fin des années 1960 (Taylor et Ramos éds. 1979 :113-115).

Dans les régions où l'impact des activités minières clandestines a été le plus intense, notamment au cœur des hautes terres de la Serra Parima, les communautés yanomami ont commencé à connaître, dès la fin des années 1980, des changements sociaux et économiques importants. Durement affectées sur le plan démographique et environnemental, elles ont opéré une série de réaménagements de leur modèle traditionnel d'occupation de l'espace forestier,

\footnotetext{
* Anthropologue, Directeur de Recherche IRD associé à l’Instituto Socioambiental (ISA) de São Paulo, Brésil.

** Géographe, Chargé de Recherche CNRS accueilli à l'IRD et affecté au Centre de Développement durable de l'Université de Brasilia (CDS-UnB).

${ }^{1}$ Recensement de la Fondation Nationale de Santé (FUNASA), octobre 2003.

2 Selon la Constitution brésilienne de 1988 (article 231).

${ }^{3}$ Dont, par exemple, T. Koch-Grünberg, H. Rice, G. Salathé, D . Holdridge, ou, plus tard, A. Gheerbrandt.

${ }^{4}$ Sur l'histoire du contact des Yanomami du Brésil, voir Albert et Kopenawa 2003 : 163-183.

${ }^{5}$ La Perimetral Norte devait être une réplique de la route Transamazonienne sur la rive gauche de l'Amazone. Le projet sera abandonné en 1976, après l'ouverture de quelques tronçons.

${ }^{6}$ Projet d'occupation de la zone frontalière nord amazonienne initié en 1985.
} 
modulés en fonction de la présence successive des chercheurs d'or puis de la structure d'assistance installée durant le processus de leur expulsion (administrations spécialisées : FNS, FUNAI et $\mathrm{ONG}^{7}$ ). Le présent travail propose l'analyse d'un exemple de cette géométrie variable des stratégies spatiales yanomami face au contact, ceci à partir de l'étude fine de la région du haut rio Mucajaí dite "Homoxi » (Figure 1), occupée par trois communautés (ensembles de maisons collectives): Tirei, Xere $u$ et Yaritha, totalisant, à l'époque de l'enquête de terrain (avril 2002) quelques 360 personnes.

A cette fin, nous décrivons dans un premier temps le contexte économique et géopolitique régional, en particulier la ruée vers l'or dans l'état de Roraima à la fin des années 1980. Ces événements, qui sont peu documentés dans la littérature scientifique, ont profondément affecté les Yanomami et leur société et sont les causes directes d'adaptations économiques et territoriales de nombreux groupes locaux qui les ont subis. Changeant d'échelle, nous étudions ensuite l'histoire des trois communautés de la région de Homoxi et le processus de transformation de leur occupation de l'espace, d'abord face à celui des orpailleurs qui ont envahi leurs terres, et, ensuite, après l'éviction de ces derniers, face à la structure permanente d'assistance locale (FNS, FUNAI).

\section{L'ouest du Roraima : zone d'exploitation minière ou aire protégée ?}

Dès sa révélation, au début des années 1970, le potentiel minier considérable de l'ouest du Roraima (Serra Parima), a mobilisé un jeu complexe d'intérêts incluant grandes entreprises du secteur, hommes politiques locaux et petits entrepreneurs d'orpaillage. Parallèlement, une meilleure connaissance anthropologique des Indiens Yanomami puis la constitution d'un réseau d'ONG indigénistes défendant leurs droits territoriaux ont suscité l'émergence d'une vaste campagne nationale pour la création d'une aire protégée. Alternant succès et reculs, les deux camps se livreront une lutte sévère durant près de deux décennies, tandis que les Yanomami verront leur population gravement affectée par une succession d'épidémies. Finalement, le contexte culturel et politique international évoluant en faveur du droit des minorités et de la préservation de l'environnement, les terres yanomami seront légalement reconnues à titre d’aire protégée au début des années 1990.

\section{Du Projeto RADAM à la ruée ver l'or du Roraima (1975-1987)}

\subsection{Surucucus : révélation du potentiel minier du territoire yanomami}

Les richesses minérales du centre du territoire yanomami (Serra Parima) ont été identifiées au milieu des années 1970, dans le cadre d'une grande opération de cartographie lancée par le gouvernement militaire de l'époque sous le nom de Projeto RADAM (voir MacMillan 1995: 24-26, Le Tourneau 1999) afin d'évaluer les ressources, en particulier minières, de l'Amazonie. De nombreux gisements potentiels (or, cassitérite ${ }^{8}$, manganèse, uranium, etc.) furent ainsi identifiés, de même que la possibilité que certains métaux, arrachés par l'érosion, se retrouvent en abondance dans les dépôts alluviaux de la région.

Publiés à une époque où le Brésil prétendait faciliter son développement par une exploitation intensive de ses ressources naturelles, les résultats du projet RADAM ont amené le gouvernement à envisager une exploitation industrielle des gisements de la Serra Parima à des fins d'exportation. Toutefois, dès mars 1975 - soit à peine un mois après l'annonce des résultats du projet RADAM -, un premier contingent de prospecteurs artisanaux de cassitérite

\footnotetext{
${ }^{7}$ Installation, depuis 1990, de postes de la Fondation Nationale de l'Indien (FUNAI), de la Fondation Nationale de Santé (FNS devenue FUNASA) puis d’ONG (Médecins du Monde puis Urihi Saúde Yanomami).

${ }^{8}$ Oxyde d'étain naturel.
} 
envahit le sud du plateau de Surucucus, au cœur du territoire yanomami. En janvier 1976, ils sont près de 500, sous contrôle d'une petite entreprise locale (Além-Equador), produisant de grandes quantités de minerai, acheminées à Boa Vista par avion à partir d'une piste locale (CCPY 1989 :7-9, Taylor et Ramos éds., 1979 :53-58).

A la suite de conflits avec les Indiens Yanomami, le gouvernement interdit en février 1976 l'accès de la zone à ces prospecteurs clandestins ${ }^{9}$. En parallèle, et alors que les demandes affluent ${ }^{10}$, il autorise successivement deux grandes compagnies minières à prospecter la région : entre novembre 1975 et février 1976 l’ICOMI (Indústria e Comércio de Minérios) ${ }^{11}$ y évalue un gisement de manganèse (Taylor et Ramos op. cit.: 62-63), alors qu'en avril 1978, la DOCEGEO (Rio Doce Geologia, filiale de la grande compagnie minière Vale do Rio Doce ou CVRD) ${ }^{12}$ ouvre un bureau à Boa Vista et commence, en mars 1979, à s'intéresser au minerai d'étain.

Aucune de ces deux tentatives ne débouchera sur une exploitation effective pour plusieurs raisons. En premier lieu, les difficultés logistiques sont considérables car les sites se situent en pleine forêt tropicale, dans une région montagneuse, à plus de 200 kilomètres d'un quelconque réseau routier et à plusieurs milliers de kilomètres du premier port. De plus, dans le cas du minerai d'étain, un autre gisement d'importance est, à l'époque, sur le point d'être mis en exploitation à proximité de Manaus ${ }^{13}$, relativisant l'intérêt économique des quelques 15000 tonnes potentielles du gisement de Surucucus (MacMillan 1995: 27), ce qui conduisit la DOCEGEO à recommander au DNPM que la région soit classée comme « réserve minérale nationale ». Enfin, la difficulté des relations avec un groupe amérindien isolé aux effectifs très nombreux (74 villages présents dans un rayon de $65 \mathrm{~km}$ de la zone des gisements de Surucucus) et la forte probabilité de voir la zone classée comme aire protégée («terre indigène »), contribuèrent à dissuader les grandes compagnies minières.

Toutefois, localement, les hommes politiques et quelques entrepreneurs ne désarment pas. Ils intriguent pour que la CVRD soit obligée de céder sa concession à une entreprise contrôlée par le gouvernement de l'état de Roraima, la CODESAIMA (Companhia de Desenvolvimento de Roraima (CCPY 1989: 10-11). En 1983, celle-ci reçoit du DNPM une concession de prospection et d'exploitation du minerai d'étain de Surucucus, pour un délai d'un an renouvelable. Elle doit, cependant, obtenir un vote favorable du Congrès National, puisque cette concession se trouve englobée dans un vaste périmètre auquel la FUNAI venait d’interdire l'accès (1982), à titre de préalable de sa légalisation au titre de " terre indigène ». Afin d'emporter l'adhésion, le projet de la CODESAIMA prétendait prendre en compte les intérêts amérindiens en proposant l'emploi de main d'œuvre yanomami et l'attribution de 5\% de royalties à la FUNAI. Présenté par un député du Roraima, un projet de loi ad hoc sera finalement repoussé en octobre 1985 du fait de la mobilisation d'associations - au premier rang desquelles la Commissão Pró-Yanomami (CCPY) ${ }^{14}$-, et de parlementaires favorables à la défense des ethnies minoritaires.

A la suite de cet échec, un ultime coup de force sera tenté par un aventurier local, José Altino Machado, entrepreneur minier amazonien, propriétaire d'une compagnie d'avion taxi et orateur charismatique. Venu du célèbre placer de la Serra Pelada (état du Pará), il fonde le

\footnotetext{
${ }^{9}$ Ordonnance 422 du 2/09/1976 du Ministère de l’intérieur (Ramos et Taylor éds. 1979: 61-62).

${ }^{10}$ Presque $60 \%$ de la superficie de cette région fait l'objet de demandes de prospection minière enregistrées au DNPM (Departamento Nacional de Produção Mineral) par des entreprises publiques et privées, nationales ou internationales (Ricardo éd. 1999: 50, 84).

${ }^{11}$ Une joint venture de la Bethlehem Steel et de la CAEMI (Companhia Auxiliar de Empresas de Mineração), qui exploite depuis 1950 le gisement de manganèse de la Serra do Navio (Amapá).

${ }^{12}$ La CVRD, très importante compagnie minière, était alors une entreprise publique. Sur la prospection de la DOCEGEO et ses suites voir CCPY 1989 : 10-15.

${ }^{13}$ Il s'agit du gisement de Pitinga, dont l'exploitation déclenchera un conflit avec les Indiens Waimiri-Atroari.

${ }^{14}$ Fondée en 1978 par un petit groupe d'anthropologues et d'indigénistes travaillant avec les Yanomami du Brésil depuis le début des années 1970.
} 
2 février 1985 l'Association des prospecteurs artisanaux de Roraima (Associação dos Faiscadores e Garimpeiros do Território Federal de Roraima) dont le but est d'exercer une pression politique pour obtenir l'accès aux zones interdites par la FUNAI. Le 14 février, il organise avec cinq avions monomoteurs et une soixantaine d'hommes armés une invasion para-militaire de la région de Surucucus, comptant sur des complicités politiques locales. Cette invasion sera repoussée grâce à l'action conjuguée du Ministère de la Justice et de la Police fédérale (PF), de nouveau sous la pression d'une campagne de la CCPY (CCPY op. cit. : 17-22).

José Altino Machado se lancera, malgré ses démêlés judiciaires, dans une active contre-offensive médiatique. Se présentant comme le porte parole d’une minorité oubliée - les chercheurs d'or (garimpeiros) - il s'en prendra alors autant aux associations pro-indiennes qu'aux grandes compagnies minières, accusées les unes comme les autres de spolier les richesses du Brésil au profit de l'étranger. Il s'appuiera alors sur les milliers de garimpeiros qui afflueront en Roraima à partir de 1985, attirés par l'or de la Serra Parima, pour affaiblir la position pro-indienne de l'État fédéral et le placer devant le fait accompli d'une invasion massive du territoire yanomami. Il sera ainsi en mesure d'accéder, cette fois impunément, au gisement de cassitérite de Surucucus qu'il convoitait et exploitera à son profit jusqu'en 1990.

\subsection{Les prémisses d'une ruée vers l'or}

Parallèlement à la série d'évènements qui agitent Surucucus, deux autres régions du territoire yanomami se voient envahies par des chercheurs d'or selon un modèle classique en Amazonie brésilienne. Ainsi, si les grandes compagnies minières ou les projets publics sont soumis à de lourdes contraintes légales, les garimpeiros, du fait de la souplesse de leurs techniques et de leur clandestinité, peuvent, au contraire, s'infiltrer très rapidement et massivement dans n'importe quelle région du pays, d'où ils ne peuvent être délogés que par une action à grande échelle du gouvernement fédéral. Dans le cas du Roraima, la motivation s'avère d'autant plus forte que le cours international de l'or était parvenu, depuis 1979, à 850 US\$ l'once (31,1 g) alors qu'il avait oscillé autour 42 US\$ de 1943 à 1973.

La première invasion a lieu au début des années 1980, avec la découverte de placers particulièrement productifs dans un ancien site exploité depuis les années 1960 sur le bas rio Uraricaá : le garimpo Santa Rosa ${ }^{15}$. Cette découverte fait affluer rapidement quelques 5000 orpailleurs dans le nord-est du territoire yanomami, soutenus par la CODESAIMA - qui révèle ainsi déjà son rôle dans l'encadrement et l'officialisation de la prospection clandestine. Début 1983, une intervention de la FUNAI donne un certain répit à la région, mais à peine six mois plus tard les placers de Santa Rosa sont à nouveau repris par 3800 orpailleurs. Dans les trois ans qui suivent, leur avancée s’étendra dans tout le bassin du haut rio Uraricaá. ${ }^{16}$

A la même époque, un autre site d'orpaillage, Apiaú velho, est également ouvert au sud-est du territoire yanomami, entre le haut rio Apiaú et le haut rio Catrimani (CCPY op. cit. : 37-44). Jusqu'en 1983 son occupation demeure assez marginale, ne rassemblant que 300 prospecteurs. Dès l'année suivante des contingents d'orpailleurs venus de différents états amazoniens (Rondônia et Pará notamment), commencent à affluer. En janvier 1985, devant l'inaction de la FUNAI, une cinquantaine de guerriers yanomami de régions voisines tente d'expulser ces chercheurs d'or, sans succès. En février, un autre groupe yanomami, cette fois accompagné de policiers militaires et fédéraux réussit à faire évacuer une centaine d'orpailleurs mais la plupart demeure sur place. La FUNAI établit alors un «poste de surveillance » sur le rio Apiaú, doté d'un détachement de cinq agents de la police militaire du Roraima. L'invasion se poursuit néanmoins et un nombre croissant d'orpailleurs s'infiltrent

\footnotetext{
${ }^{15}$ Affluent de rive gauche du rio Uraricoera, un des formateurs principaux du Rio Branco.

${ }^{16}$ Sur la pénétration des orpailleurs dans cette région voir CCPY op. cit. : 24-35 et Ramos et al. 1987.
} 
par petits groupes le long des rio Mucajaí et Apiaú, contournant le poste de la FUNAI. En juillet 1985, un nouveau site aurifère est en opération sur le rio Novo, affluent de l'Apiaú, les chercheurs d'or étant cette fois évalués à plus de 600. Parlementaires, commerçants et entrepreneurs de transport aérien locaux s'engagent alors activement dans la campagne du syndicat de garimpeiros de José Altino en faveur de la légalisation de l'orpaillage en territoire yanomami. A la fin 1985 l'effectif des orpailleurs de l'Apiaú a doublé. La FUNAI et la police militaire, submergées, ont abandonné leur poste. En 1986 la découverte d'un nouveau placer important aux sources de l'Apiaú (Cambalacho) augmentera encore le nombre des garimpeiros dans la région (MacMillan 1995 :30).

Au plan national, la réputation de richesse aurifère du territoire yanomami ne cesse de croître, dans un contexte amazonien où l'orpaillage est devenu une activité économique majeure (Cleary 1990). Ce fait ne semble pas contrarier les autorités fédérales, sous influence de l'armée, toujours puissante (Albert 1987), qui a décidé de promouvoir une occupation militaire et économique de ce qu'elle tient pour le « vide » amazonien par le biais du Projeto Calha Norte (PCN). Confrontés au processus d'occupation accélérée de l'ouest du Roraima, les généraux semblent considérer plus intéressant d'y favoriser la présence des chercheurs d’or (« véritables » brésiliens), que de réserver une grande quantité de terres aux Yanomami, considérés peu ou prou comme d'inutiles et dangereux apatrides (Albert 1990, 1992). Toutes les conditions pour le déclenchement d'une ruée vers l'or de grande ampleur étaient ainsi réunies.

\section{1987-1990 : la ruée vers l'or}

\subsection{Far west amazonien}

En juillet 1987 les orpailleurs du cours supérieur du rio Apiaú parviennent sur un affluent du haut rio Mucajaí, le Couto de Magalhães, y découvrent un nouveau site aurifère important (Novo Cruzado) et y assassinent quatre leaders yanomami qui tentent d'entraver leur avancée. La répercussion médiatique de ces meurtres entraîne le déclenchement d'une opération militaire qui évacue, pour la forme, quelques centaines d'orpailleurs mais, surtout, expulse au même titre ONG et missions de la région au prétexte qu'il faut "retirer tous les éléments étrangers qui perturbent la vie des Indiens ». Dans les faits, et vu le peu de zèle que le gouvernement mettra à empêcher les orpailleurs de retourner à leurs placers, cet événement paraît confirmer la volonté de favoriser une situation d'invasion qui constitue un fait accompli à même de provoquer une révision du périmètre accordé aux Yanomami, en plein accord avec les objectifs du PCN (Ramos 1998: 227-42). Comme on peut l'imaginer, les chercheurs d'or voient dans la décision des autorités le signe de leur complicité et débordent rapidement le maigre dispositif mis en place pour les contenir.

Un flux de plus en plus considérable d'orpailleurs se dissémine dans le bassin du haut Mucajaí : ils étaient déjà plus de 5000 en décembre 1987 autour de la piste de Paapiú et les effectifs augmentent de manière exponentielle au fur et à mesure que la rumeur (fofoca) de la richesse des placers du territoire yanomami se propage dans le monde du garimpo. La population locale elle-même est prise par la fièvre de l'or. On trouve parmi les orpailleurs, hormis un contingent de professionnels, une grande majorité de petits agriculteurs, mais aussi des pompiers, policiers, employés, conseillers municipaux, maires, avocats, médecins qui abandonnent leur emploi, de façon temporaire ou permanente, pour les placers des terres yanomami. Quelques $130 \mathrm{~kg}$ d'or sont commercialisés par mois à Boa Vista. En décembre 1987, le Président de la FUNAI et le gouverneur du Roraima proclament la légalisation de "réserves d'orpaillage » au sein du territoire yanomami (CCPY 1989 : 46-58) et dès janvier 1988, les chercheurs d'or sont estimés à 10 000. La piste de Paapiú est devenue la plaque 
tournante des garimpo de la région. On estime à 130 les vols qui décollent chaque jour de Boa Vista pour l'approvisionner (CCPY op. cit. :59).

La prise de possession de cette infrastructure par un lieutenant de la Police militaire chargé de sa surveillance (fin 1987), associé à un groupe d'orpailleurs armés ${ }^{17}$, a permis le développement à grande échelle de l'orpaillage dans ses alentours. La zone aurifère, d'une exceptionnelle richesse, se situe le long des fleuves descendant de la Serra Parima et, en particulier, des hauts rios Mucajaí, Parima et Catrimani. Il s’agit de sites très isolés : pour les atteindre depuis Boa Vista il faut traverser, en moyenne, plus de 150 kilomètres de forêt tropicale dense et accidentée. Si l'approvisionnement terrestre est impossible, le ravitaillement en avion-taxi n'est pas non plus très aisé, car le coût des trajets est très élevé. Elargie et agrandie par l'armée de l'air (800 mètres de long), et donc en mesure de recevoir des avions lourds comme des DC-3, la piste de Paapiú est rapidement devenue une tête de pont irremplaçable. Les entreprises de taxi aérien y basent des hélicoptères ; des équipements lourds et encombrants (motopompes, fûts de carburant, conduites) peuvent également y être acheminés. Grâce au soutien de cette base avancée, les prospecteurs rayonnent sans difficulté dans la région et y ouvrent des placers de plus en plus nombreux ainsi que des nouveaux terrains d'aviation attenants aux plus importants. L’or alluvial est le métal le plus recherché, mais on note également une exploitation artisanale importante des affleurements de cassitérite du haut Parima (région de Xitei, au sud de Surucucus). ${ }^{18}$

En 1989 de 30 à 40000 personnes travaillent dans les placers du territoire yanomami (MacMillan 1995: 31) et selon une étude du gouvernement de Roraima, 66000 personnes vivent directement ou indirectement de cette activité (CCPY op. cit. :69). Le cours supérieur du rio Mucajaí constitue l'épicentre de cette ruée vers l'or: la majorité des 73 pistes clandestines du territoire yanomami s'y trouvent (voir Figure 2 et MacMillan op. cit.: 38-39). Entre 1988 et 1990 la production d’or du Roraima est estimée à 49 tonnes (ibid. : 34).

\subsection{Les Yanomami encerclés}

De 1987 au début 1990 les Yanomami de l'état de Roraima ont vécu littéralement encerclés dans leurs maisons collectives par une population d'orpailleurs représentant environ six fois leur propre effectif. Cette invasion massive a eu un impact épidémiologique et écologique considérable, à bien des titres beaucoup plus grave que celui des projets routiers et agricoles qui les avaient affectés dans les années 1970. Les épidémies de paludisme et d'infections respiratoires, ajoutées aux conflits armés sporadiques, ont causés la disparition d'environ $13 \%$ de la population yanomami du Brésil durant cette période. ${ }^{19}$ Par ailleurs, le bouleversement et la pollution du lit des rivières ont entraîné des dommages importants et durables à l'environnement forestier et fluvial, base du système productif de ces indiens.

Dans ce contexte, et selon le scénario qui se dessinait depuis le début de l’invasion, tant les parlementaires locaux que les responsables politiques nationaux proposeront d'altérer les limites des terres yanomami afin d'entériner la présence massive des orpailleurs. Le gouvernement promulguera ainsi en 1989 une série de décrets réduisant la « Terre Indigène Yanomami » interdite en 1982 à 19 micro-réserves dont la superficie cumulée ne représente que moins de deux tiers du total, et dont le dessin permettrait aux principaux sites aurifères de se trouver dans des zones de "Forêt nationales ", unités de conservation dont le réglement devait être modifié pour permettre cette activité (Albert 1992, Albert et Le Tourneau 2004).

\footnotetext{
${ }^{17}$ Voir, sur cet épisode, MacMillan 1995:36-37.

${ }_{18}^{18}$ Notamment par José Altino Machado (MacMillan 1995: 45-48).

${ }^{19}$ Projection à partir des données de APC 1990: 32. Un rapport du Ministère brésilien de la Santé (1990, Projeto Saúde Yanomami) évaluait officiellement ces morts à " plus de mille ". Sur cette situation sanitaire catastrophique voir également CCPY 1989: 60-71, Albert 1990 et Pithan et al. 1991.
} 
Par ailleurs, trois "Réserves d'orpaillage » sont créées (1990) au sein de l'ensemble, légalisant ainsi la présence et l'activité des chercheurs d'or dans les zones les plus envahies. Enfin, sur le plan politique local, R. Jucá, président contesté de la FUNAI depuis 1986 en raison de ses positions favorables à la réduction du territoire yanomami, se voit nommer gouverneur de l'état de Roraima. Il s'efforce alors d'entamer un processus de légalisation des activités d'orpaillage dans la région via la CODESAIMA, sur le même modèle que celui de l'encadrement du garimpo Santa Rosa.

Toutefois, de manière inattendue pour les politiciens et prospecteurs locaux - aveugles aux mutations globalisées de la fin des années 1980 - la médiatisation à grande échelle de la tragédie sanitaire et écologique yanomami et une vaste campagne internationale menée par les ONG (de plus en plus relayée par l'opposition politique brésilienne puis par les organisations multilatérales et certains gouvernements étrangers) renverseront le rapport de force local très défavorable aux Indiens. Devant ce qui était devenu unanimement, dans la presse internationale, « le génocide des Yanomami » et dans un contexte de montée en puissance des préoccupations écologiques internationales - qui aboutira au Sommet de la Terre de Rio en 1992 - manifestations publiques, pressions politiques et campagnes de presse créent peu à peu un climat extrêmement négatif pour le Brésil.

\subsection{Reconnaissance de la Terre Indigène Yanomami : fin de partie ?}

Le Président F. Collor, qui prend ses fonctions en janvier 1990, se trouve contraint de redorer l'image du pays à l'étranger et de regagner la confiance des grands bailleurs de fonds internationaux qui lient de plus en plus leurs prêts à des conditions de protection des minorités ethniques et des milieux naturels. Dès son entrée en fonction, il lance une campagne, très médiatique, de "sauvetage" des Yanomami, baptisée Selva Livre. Des opérations d'évacuation des orpailleurs par la PF sont organisées, un plan de secours médical d'urgence est mis en place, les « réserves d'orpaillage » sont annulées, et, enfin, en mai 1990, treize "pistes clandestines » sont dynamitées devant les caméras de la TV Globo et des photographes de la grande presse (sur 73 officiellement recensées).

Ce volontarisme publicitaire trouva rapidement ses limites, tant du fait du manque d'organisation des institutions chargées de l'expulsion des orpailleurs que du caractère ponctuel des opérations. Quelques mois après le passage de la PF, bien des pistes sont réparées et remises en service, tandis que celles qui n’ont pas été endommagées continuent à opérer. ${ }^{20}$ En fait, les orpailleurs occasionnels sont les premières « victimes » de ces opérations d'expulsion alors que les professionnels, demeurés sur place, tirent un certain bénéfice de la mise à mal du système de tribut qui les soumettait aux patrons de pistes ou de placer $^{21}$.

Fin 1990, une nouvelle opération est engagée par la PF, cette fois mieux organisée et assortie d'une destruction systématique des campements et du matériel des orpailleurs. Ce nouvel épisode signale le début d'une période dans laquelle l'ouverture et l'exploitation de nouveaux placers devient de plus en plus coûteuse et hasardeuse dans la mesure où se trouve grippé le processus de réinvestissement rapide des gains dans la prospection et les nouveaux équipements (MacMillan op. cit. 1995:52). Par ailleurs, les postes mis en place par la Fondation Nationale de Santé (FNS) pour assister les Indiens exercent une certaine surveillance du territoire et, même si les opérations d'expulsion et de destruction de matériel ne sont que sporadiques, elles font peser une menace qui ralentit l’activité en décourageant les

\footnotetext{
${ }^{20}$ Dès la fin mai 19905000 orpailleurs étaient de retour sur le territoire yanomami, sans compter les 8000 qui continuaient à opérer dans les « réserves d'orpaillages » créées par le gouvernement précédent (Albert 1992). Les opérations de retrait des garimpeiros reprendront quelques mois après et se poursuivront, de façon sporadique, au cours de toute la décennie.

${ }^{21}$ Voir, sur ce point, MacMillan 1995 : 51-52 et Geffray 1995: 68-70.
} 
investissements qui, par ailleurs, pâtissent d'une baisse progressive du cours de l'or dans les années 1990.

Malgré une grave rechute en $1993,{ }^{22}$ la ruée vers l'or en territoire yanomami se verra ainsi peu à peu endiguée par cette suite d'opérations de la FUNAI et de la PF.Toutefois, le changement le plus important s'est joué sur le plan législatif. En signant, en mai 1992, un décret qui homologue les limites du territoire yanomami, le président Collor a tranché le débat ouvert dès 1968 à propos du périmètre exact de la zone qui devait être dévolue aux Indiens. Reconnaissant le bien fondé des rapports anthropologiques établis dès 1978 et leur conformité avec les principes de la Constitution, ce décret considère comme "Terre Indigène Yanomami » une grande part des terres de l'ouest du Roraima (et la rive gauche du Rio Negro jusqu'au Cauaboris), revenant sur le démembrement en 19 " îles » édicté en 1989. Il donne de ce fait un socle solide tant à l'action du pouvoir exécutif qu'au lobby indigéniste : une fois reconnu au terres yanomami le caractère de "terre indigène " (alors que depuis 1982 il ne s'agissait que d'une « zone interdite pour identification »), s'ensuit logiquement que la loi doit s'y appliquer et donc que l'occupation des orpailleurs ne se situe plus dans les limbes juridiques, mais nettement en infraction avec les dispositions de la Constitution et de la législation indigéniste nationale.

Ce nouveau contexte n'empêchera toutefois pas que des petits groupes d'orpailleurs cherchent à revenir sur les lieux et à tenter de reprendre leurs activités durant toute la décennie, en particulier à chaque fois que le cours de l'or s'oriente à la hausse. De telles « réinvasions ", bien que limitées, sont continuelles depuis 1992 et entraînent toujours les mêmes conséquences : violences - dont le massacre barbare de 16 Yanomami en 1993 à Haximu (Albert 1994) - et contamination (de 1991 à 1998 sont morts 1211 Indiens, la plupart de paludisme et d'infections respiratoires). ${ }^{23}$ Cinq foyers d'orpaillage étaient encore en activité sur le territoire Yanomami en 2003, pour un total estimé de 200 garimpeiros. $^{24}$

\subsection{Assistance sanitaire et éducation interculturelle : une nouvelle relation avec les Blancs}

En dépit de cette présence résiduelle, une nouvelle époque s'est ouverte dans les années 1990 pour les Yanomami du Brésil, dans laquelle plusieurs facteurs convergents ont favorisé un sursaut démographique et politique. Le gouvernement brésilien a confié en 1999 l'assistance sanitaire yanomami à plusieurs ONG dans le cadre d'un «District sanitaire indigène spécial » (DSEI), sous son contrôle et bénéficiant d'un important financement public. Cette initiative s'est révélée très positive, ainsi les indices d'infestation palustre (pour mille habitants) sont-ils passés, chez les Yanomami de l'état de Roraima, de 616,77 en 1998 à 11,74 en 2002. Le coefficient de mortalité (pour mille habitants) a chuté de 22,9 en 1998 à 3 en 2002, tandis que le taux de croissance démographique annuel depuis 2000 est de $4,5 \%{ }^{25}$

Par ailleurs, ONG et organisations missionnaires ont mis en place dans la région, depuis la moitié des années 1990, divers programmes d'éducation bilingues et de formation interculturelle. Ces programmes, ainsi que la participation active des Indiens aux Conseils du DSEI Yanomami, ont favorisé, en une décennie, l'émergence d'une génération de jeunes professeurs et représentants yanomami qui viennent prolonger l'action de certains leaders historiques, tel Davi Kopenawa. L'amplification de cette dynamique a favorisé le développement progressif d'une conscience collective fondée sur la défense de droits

\footnotetext{
${ }^{22}$ Entre 1992 et 1993 le nombre des orpailleurs en territoire yanomami repassera de 2000 à 11000 (MacMillan 1995 :52).

${ }^{23}$ Rapport du secteur d'épidémiologie de la FUNASA-Roraima, juin 1998.

${ }^{24}$ Rapports FUNAI (Boa Vista-Roraima), août 2002 et mars 2003.

${ }^{25}$ Rapport d'avril 2003 de l'ONG Urihi Saúde Yanomami qui a apporté une assistance sanitaire à la majorité des Yanomami de l'état de Roraima de fin 1999 à la mi 2004, date à laquelle la FUNASA a repris la gestion directe des services de santé destinés aux Yanomami.
} 
territoriaux, civils et culturels reconnus par la Constitution brésilienne, conscience qui soustend une croissante organisation politique des Yanomami sur la scène régionale ${ }^{26}$.

Cet essor démographique et cette "reprise d'initiative » ethnopolitique ne doivent cependant pas laisser oublier que, pour de nombreuses communautés yanomami, les bouleversements sociaux et les dégâts écologiques occasionnés par la présence des orpailleurs se font toujours sentir lourdement, près de quinze ans après le début de leur expulsion officielle. C'est le cas des villages de la région de Homoxi (Tirei, Xere u et Yaritha) dont nous allons maintenant examiner la situation, depuis leur première rencontre avec les garimpeiros, en 1988, jusqu’à avril 2002, date de notre première étude de terrain.

\section{De la Pista Jeremias au Posto Homoxi: la ruée vers l’or sur le haut rio Mucajaí et ses conséquences pour les communautés yanomami}

Le cours supérieur du rio Mucajaí, territoire traditionnel d'un ensemble de groupes yanomami, est l'une des régions les plus affectées par l'action des chercheurs d'or à la fin des années 1980, attirant sur une portion d'une vingtaine de kilomètres plusieurs milliers d'entre eux et voyant l'ouverture rapide de plusieurs agrégats de placers, chacun pourvu d'un terrain d'aviation de fortune (Figure 3). Dans la région dite "Homoxi », objet de la présente étude, l'invasion commença dès 1988 avec l'installation d'une piste de terre battue de 700 mètres baptisée Pista Jeremias . Ses entours feront l'objet d'une intense exploitation de la part des orpailleurs jusqu'aux premières opérations d'expulsion menées à partir de janvier 1990. Durant ce laps de temps, les communautés yanomami installées dans la zone ont été soumises à un contact intense avec les garimpeiros, entraînant de graves conséquences sanitaires et sociales qui expliquent en partie leur localisation et leur mode de vie actuels.

Contrairement aux autres pistes ouvertes dans la région, Jeremias ne sera pas abandonnée après le rush aurifère. Du fait de sa taille et de sa localisation, qui la rendent plus sûre, elle sera utilisée par les postes avancés des administrations intervenant dans la zone durant et après l'expulsion des chercheurs d'or. Elle abritait au moment de notre étude un poste de la FUNAI et un poste de santé de l'ONG Urihi Saúde Yanomami, l'endroit ayant été rebaptisé « Posto Homoxi » en raison de la proximité d'un petit affluent de la rive droite du haut Mucajaí situé en amont et nommé Hoomoxi $u$ en Yanomami. La population indienne assistée par ces deux postes se répartissait (avril 2002) en trois ensemble de maisons collectives (yano ou xapono) : Tirei, Xere u et Yaritha pour un total de 359 personnes.

Pour comprendre les implications socioéconomiques de la ruée vers l’or dans la région, nous présenterons l'histoire récente de ces trois groupes, nous focalisant particulièrement sur les Tirei, plus affectés que les autres dans la mesure où ils ont tenté de demeurer dans la région immédiate de l'orpaillage intensif en tentant d'adapter leur système productif traditionnel aux lourdes contraintes imposées par cette situation de cohabitation forcée.

\section{Les Yanomami de la région du haut rio Mucajaí}

\section{Le groupe de Tirei}

Les Tirei (85 personnes en avril 2002) occupent actuellement une maison collective unique formant un auvent circulaire d'environ 70 mètres de diamètre, située à proximité du Poste Homoxi. Le manque de feuilles de couverture (Geonoma sp.) et de ressources alimentaires dans la région (rareté de la chasse et taille réduite des jardins) font que la

\footnotetext{
${ }^{26}$ Cette dynamique débouchera sur la création d'une association yanomami en novembre 2004 (Hutukara Associação Yanomami).
} 
construction de cette maison, entreprise en septembre 2001 n'était toujours pas achevée en avril 2002. Des matériaux « exotiques » comme des bâches de plastique bleu ciel ou des tôles provenant de carcasses d'avions accidentés sont utilisées en guise de toit ou de parois, rappelant le «temps des orpailleurs » (karipiri tëhë). La construction d'une maison de ce type et de cette taille constitue un changement important pour ce groupe, peut-être influencé par les voyages de leur leader dans d'autres villages yanomami des basses terres. Avant 2001 en effet, les Tirei ont toujours constitué un ensemble de trois ou quatre petites maisons collectives proches, de forme tronconique, selon un modèle socio-résidentiel habituel dans les hautes terres yanomami.

La mémoire du groupe fait remonter son origine au sud de la montagne Hëëmakí, dans la région de Xitei (lieu-dit Yanaema, probablement occupé dans les années 50). Son nom vient d'un site postérieur (Tirei, probablement occupé dans les années 70) situé près de la rivière Kuneamari $u$, affluent du haut Parima (Figure 4). Durant la période de migration entre Yanaema e Tirei plusieurs autres sites furent plus brièvement occupés, lors d'une période d'affrontement guerrier avec des groupes de la région de Xitei et avec les habitants de Hara $u^{27}$. Après Tirei, le groupe, toujours en guerre, se déplacera vers le cours supérieur du rio Parima, à Potomatha, entre les sources du Kuneamari u et celles du Kurema u (ou Haxi u), établissant à cet endroit trois maisons collectives proches. Plus tard, il s'installera sur le haut Thoothothopi $u$, affluent du haut Mucajaí qui débouche au sud de la piste du Poste Homoxi (années 80), cette fois subdivisé en cinq maisons. Finalement, en raison de la rareté en poissons dans cette rivière, les Tirei s’établiront sur la berge du cours supérieur du Mucajaí (Ixоа и ou Uxиа и).

A l'arrivée des orpailleurs, les Tirei habitaient quatre habitations collectives: trois sur la partie inférieure du Narahipi $u$, affluent de la rive gauche du haut Mucajaí, et une sur le bas Wakapoko $u$, tributaire de sa rive droite. Cette dernière sera rapidement abandonnée car les orpailleurs ouvrirent à cet endroit une piste baptisée Macarrão, détruisant une partie des jardins yanomami. Bien que s’étant retrouvés involontairement au cœur de la zone d'orpaillage, les Tirei demeureront dans leurs maisons du Narahipi u jusqu'au début des années 1990. Ils décident alors d'abandonner les alentours de la piste Macarrão, non seulement afin de s’éloigner du cours supérieur du Mucajaí, bouleversé et pollué, mais surtout pour échapper au paludisme devenu épidémique dans cette région peu après l'expulsion du gros des chercheurs d'or ${ }^{28}$. D'abord réfugié sur le site de Xere $u$, le groupe entame alors une série de micro-migrations en direction du nouveau poste de la FUNAI et de la FNS établi auprès de la piste Jeremias (futur Posto Homoxi), avant tout attiré par les perspectives de soins médicaux et de troc (nourritures, outils, vêtements, etc.) que ce dernier offrait, à un moment où la disparition des orpailleurs avait interrompu leur accès aux bien industrialisés.

\section{Le groupe de Xere $u$}

Les Xereu sont situés au sud est du Poste Homoxi, à environ 7 km à vol d'oiseau. Leur population se répartit en deux habitations collectives coniques de 44 et 40 habitants, situés le long du haut Xere u (affluent de la rive droite du haut Mucajaí) à environ 15 minutes à pied l'un de l'autre.

Les Xereu proviennent d'une scission des Tirei, dont ils se sont séparés au moment où le groupe, encore uni, s'était réfugié près de l'embouchure du Xere $u$, regroupé en trois maisons collectives, une des Tirei proprement dits et deux autres des futurs Xere $u$. La partie

\footnotetext{
${ }^{27}$ Victimes du massacre de 1993 (voir ci-dessus II. 2.3).

${ }^{28}$ Probablement à cause de la prolifération des moustiques dans les eaux dormantes des placers abandonnés mais aussi en raison de l'inefficacité de l'assistance sanitaire de la FNS à l'époque : en mai 1991, 67\% des Yanomami de la région étaient gravement impaludés (Rapport Dr. A. Alves Braz, juin 1991).
} 
du groupe qui conserva le nom de Tirei, s'éloigna du rio Xere $u$ alors que l'autre, maintenant son organisation en deux maisons, demeura dans un premier temps sur le même site. Ils connurent par la suite une succession de brèves migrations avant de parvenir à leur position actuelle. Après leur séparation d'avec les Tirei, ils se joignirent de nouveau à eux pour un temps (sur le Apiahipi $u$ ) pour se rapprocher du Poste Homoxi, avant, finalement, de choisir de retourner sur le Xere $u$, en amont de leur premier site (où les terres disponibles pour l'agriculture étaient limitées), préférant ainsi s'éloigner définitivement des zones d'orpaillages, afin, selon leurs termes, d'échapper à la « faim de gibier » et aux maladies, dans une « parcelle de forêt encore saine » (urihi temi patiopëha).

L'ensemble formé par les Tirei et les Xereu représente aujourd'hui un contingent de 169 personnes de même origine historique, distinct de l'ensemble des Yaritha, avec lequel il a maintenu des relations étroites mais conflictuelles durant (au moins) ces dernières décennies.

\section{Le groupe de Yaritha}

Les Yaritha comptent 190 personnes réparties en quatre maisons collectives tronconiques proches et cinq petites maisons adjacentes. Yaritha est une région de terres basses, giboyeuse et fertile, située en territoire vénézuélien, sur le haut Orénoque, à sept kilomètres de la frontière et à treize du Poste Homoxi (Figure 4). La migration vers Yaritha a été préparée par l'ouverture de nouveaux jardins au début de l'été 2000 (septembre-octobre). Cette migration a été essentiellement motivée par le manque de zones cultivables dans la région encaissée de Wiramapi $u$.

Les Yaritha retracent leur origine, comme les Tirei, à partir d'une communauté historique unique, située à proximité de la montagne Hëëmaki, dans la région de Xitei (site probablement occupé dans les années 60). De cet endroit, une partie des ancêtres des Yaritha actuels se sont séparés des autres ${ }^{29}$, après une période de conflits internes, pour s’installer en plusieurs sites successifs le long du Kuneamari $u$, affluent du haut Parima (années 70). Après avoir parcouru progressivement le cours de cette rivière, divisés en trois xapono, les anciens Yaritha sont passés (début des années 80) sur le bassin du Hoomoxi $u$, tributaire du haut Mucajaí situé plus à l'est. Ils eurent à cet endroit leur premier contact avec les Blancs : la visite d'agents de la FUNAI venus en hélicoptère d'un poste situé au nord, sur le plateau de Surucucus. Après plusieurs années passées dans cette région, finalement regroupés dans une seule et vaste maison collective, ils furent les victimes d'une grave épidémie d'anthrax qui provoqua plusieurs morts. Ils quittèrent alors ce site pour se réfugier en amont du Hoomoxi $u$ où ils se divisèrent à nouveau en cinq xapono. C'est là qu'ils rencontreront les orpailleurs en 1988 : en visite sur leur ancien site du bas Hoomoxi $u$, ils constatèrent que ces derniers avaient détruit leur habitation et commencé à ouvrir une piste (Julio do blefe) sur l'emplacement de leurs anciens jardins.

Comme les Tirei, les Yaritha (alors Hoomoxi) ont maintenu des relations d'échanges et de conflits avec les chercheurs d'or en 1988 et 1989. Cependant, contrairement au Tirei (mais de manière similaire aux Xere $u$ ), ils ont choisi, au début des années 90, de fuir les dégradations écologiques et les épidémies de paludisme de la zone d'orpaillage, renonçant de ce fait aux bénéfices économiques et sanitaires de la proximité directe du Poste Homoxi établi par la FUNAI et la FNS. Ils s'établirent donc en 1994 au lieu-dit Wiramapi $u$, situé dans les hautes terres du sud et loin de la zone d'orpaillage, sur un tributaire des sources de l’Orénoque, à huit kilomètres du Poste Homoxi.

\footnotetext{
${ }^{29}$ L'autre partie forme aujourd'hui le groupe de Yarakapi $u$, actuellement situés sur un affluent du haut Orénoque, au Venezuela. Ce groupe, comme celui de Hara u, situé en aval, a des relations plus distantes avec le poste Homoxi bien qu'il visite régulièrement les habitants de Yaritha.
} 
Dynamique migratoire et contexte politique régional

Les trois groupes qui occupent aujourd'hui la région de Homoxi forment donc deux ensembles - Tirei et Xereu d'une part et Yaritha de l'autre - qui ont une origine historique commune au sud de la montagne Hëëmaki (région de Xitei). Ils ont ensuite remonté le Kuneamari $u$ (haut Parima) en direction du haut Mucajaí, les premiers adoptant une trajectoire plus septentrionale (probablement avec une certaine antériorité). Ces deux ensembles ont maintenu, durant ces migrations, des relations cérémonielles (fêtes d'alliance reahu), politiques (raids guerriers communs) et socio-économiques (marriages, troc) étroites, non sans avoir également connu des conflits sporadiques. Ceux-ci se sont poursuivis (et parfois aggravés) dans la période qui a suivi le départ des orpailleurs,, dans la mesure où les Tirei ont décidé de lier leur destin au Poste Homoxi, entendant ainsi conserver le monopole sur les biens et service qui en émanaient en évitant que les autres communautés n’y aient accès.

En dehors de ces rapports réciproques, les deux ensembles entretiennent des relations historiques avec les groupes du haut Orénoque: Yarakapi $u$ et Hara $u$. Dans un cercle plus large, ils ont maintenant également des interactions sporadiques avec, au sud, les groupes du rio Toototobi (affluent du haut rio Demini) ; à l'est, avec ceux du haut Couto de Magalhães (affluent du haut Mucajaí) et au nord-ouest, avec ceux de la région de Xitei. Au nord-est, la situation est plus délicate. Après une série de conflits mineurs à propos de biens de troc, initiée en novembre 95 et qui finit par produire une mort accidentelle lors d'un duel rituel (février 96), les Tirei sont entrés en guerre avec des groupes (Kurema u et Huruna) arrivés récemment dans la région pour s'établir dans l'orbite du poste Homoxi. Un cycle de raids guerriers réciproques s'ensuivit, qui dura jusqu’à la fin 1999, causant la mort d'une dizaine de personnes de part et d'autre ${ }^{30}$.

Cet épisode guerrier récent a eu une grande influence sur la vie des Tirei dans les années qui ont suivi l'expulsion de la majorité des orpailleurs de la région, conditionnant la mobilité du groupe (qui s'astreignait à de longues périodes de nomadisme loin de son site de résidence habituel après chaque raid et évitait une série de zones "dangereuses » dans ses chasses et voyages), augmentant ainsi sa dépendance alimentaire vis-à-vis de ses alliés, en particulier des habitants de Yaritha (à l'époque Wiramapi $u$ ), et vis-à-vis du Poste Homoxi. La crainte d'une attaque surprise de la part de leurs ennemis était encore très perceptible au moment de notre étude de terrain en avril 2002

\section{De l'arrivée des chercheurs d'or sur le haut Mucajaí (1988) à l'ouverture du Poste Homoxi (1990)}

A l'arrivée des orpailleurs, les Tirei avaient quitté depuis quelques temps déjà leurs anciens jardins de Thoothothopi $u$ afin de se rapprocher des berges du haut Mucajaí (embouchure du Narahipi $u$ ), et d'avoir ainsi accès aux ressources d'un cours d'eau plus important. La région disposait également de sols fertiles et d'une forêt giboyeuse le long d'un ensemble de petites rivières proches. Le système d'usage de l'espace mis en place à l'époque correspondait au modèle classique chez les Yanomami (Albert et Gomez 1997: 34-41). Le groupe disposait ainsi de jardins en production près de leur maison principale et avait recours à un réseau quotidien d'itinéraires et de sites de chasse, de pêche et de collecte. Par ailleurs, des séjours d'assez longue durée loin de leur maison, dans des campements forestiers

\footnotetext{
${ }^{30}$ La plupart de ces morts sont dûes aux fusils de chasse obtenus auprès des orpailleurs, les raids avec arc et flèches étant bien moins meurtriers. Rapts de femmes, vols ou conflits de troc ne constituent jamais la cause directe d'une incursion guerrière yanomami. Un raid n'est motivé que par une mort à venger, souvent la victime d'un raid ennemi antérieur. Toutefois, un décès à l'origine d'un cycle guerrier peut être aussi bien attribué à des sorciers ennemis (okapë) ou avoir été provoqué accidentellement durant les duels rituels organisés pour résoudre les conflits mineurs entre alliés.
} 
(expéditions collectives de chasse et de collecte), chez des alliés (fêtes et visites) ou auprès d'anciens sites permettaient d'avoir accès à un surcroît de ressources alimentaires (cultigènes et gibier, notamment), tout en ralentissant l'inéluctable processus d'épuisement des ressources, notamment cynégétiques, autour de leur maison actuelle, processus devant mener, tôt ou tard, à une nouvelle migration.

\section{Premiers contacts}

D’après les témoignages recueillis, les premiers orpailleurs arrivèrent à pied, jusqu'à une des maisons yanomami du Narahipi $u$, sur le haut Mucajaí, rivière dont ils remontaient le cours en prospectant de nouveaux sites aurifères ${ }^{31}$. Le succès de ces recherches les amena à y établir un premier placer ravitaillé en matériel et en vivres lancés en forêt depuis des avions monomoteurs. De nouveaux orpailleurs, en plus grand nombre, vinrent se joindre peu à peu à ce premier groupe, ouvrant des clairières pour amener, par hélicoptère, des motopompes ${ }^{32}$. Puis, les résultats de cette première exploitation s'étant montrés prometteurs, ces premiers orpailleurs se retirèrent momentanément de la région pour préparer son exploitation à plus grande échelle.

L'étape suivante vit, en effet, l'ouverture de plusieurs pistes le long de la rivière, d'abord Baiano Formiga, puis Macarrão, Pau Grosso (bas Xere u), Jeremias, Julio do blefe, Chimarrão, etc. pour un total de 10 pistes ouvertes le long du haut Mucajai sur une distance d'environ vingt kilomètres (Figure 3). L'ouverture de toutes ces pistes suivra le processus décrit par MacMillan pour le premier site aurifère important du haut Mucajaí nommé Grota do Tarzã, découvert en mars 1988, où, après quelques mois, travaillaient déjà 400 orpailleurs (Mac Millan 1995: 40-43). Les placers de la région se sont ainsi déployés à partir du réseau de pistes situé le long de cette rivière, les garimpos mécanisés demeurant concentrés dans la zone riveraine tandis que les garimpos manuels fleurissaient aux alentours, sur de petits affluents, en pleine forêt.

Dans un premier temps, les Tirei (comme bien d'autres groupes yanomami) ont bien accueilli les orpailleurs, se méprenant sur leurs intentions et espérant établir avec eux des relations de cohabitation pacifique basée sur des échanges profitables. Selon leurs termes : « Nous avons manqué de sagesse en ce temps, nous pensions que les chercheurs d'or étaient de vrais étrangers (napëpë yai), des visisteurs amicaux qui souhaitaient faire du troc avec nous ». L'expression vrais étrangers se réfère ici à un mythe d'origine des Blancs (napëpë : « étrangers, ennemis »), créés à partir de l'écume du sang d'ancêtres yanomami par le démiurge Omama. Lors de leur retour dans la forêt avec les marchandises octroyées par leur créateur, ces étrangers devraient être ainsi tenus à un devoir de générosité envers les yanomami dont ils sont issus et qui sont restés sur place ${ }^{33}$.

Ainsi, les Yanomami du haut Mucajaí ont-ils commencé à échanger la production de leurs jardins avec l'avant-garde des orpailleurs, encore peu nombreuse, contre des vêtements, des hamacs, des outils métalliques, des marmites d'aluminium, des fusils et des cartouches, sans percevoir que pour les Blancs il s'agissait ainsi, dans un premier temps, de faire tolérer leur présence (Albert 1994). Toutefois, l'augmentation rapide du flux des nouveaux arrivants, les ouvertures successives de pistes, puis l'invasion massive de la région, rendirent rapidement la situation beaucoup plus menaçante. Il ne resta plus alors aux Tirei, submergés, qu’à composer avec la nouvelle réalité de leur territoire envahi.

\footnotetext{
${ }^{31}$ Leur premier campement dans la région avait été établi près de ce qui sera plus tard la piste Pau grosso.

32 La technique habituelle des orpailleurs amazoniens consiste à liquéfier les berges des rivières par des jets à haute pression puis à aspirer le limon résultant et à séparer l'or des graviers.

${ }^{33}$ Voir les mythes recueillis par B.A. in Wilbert et Simoneau 1990 : 79-81 et 82-83.
} 


\section{Une cohabitation inégale}

En dépit de situations de tensions sociales récurrentes et d'une pression croissante sur leurs ressources naturelles, les Tirei s'efforcèrent d'adapter leur usage de l'espace et leur système productif à l'occupation de la région par les orpailleurs. Ils ont ainsi abandonné leurs maisons riveraines au profit d'habitations situées sur le haut Narahipi $u$, échappant au pire des nuisances tout en maintenant avec les intrus - en dépit d'importants sacrifices (sanitaires et écologiques) - des échanges réguliers de services (transport d’eau, bois de chauffage, travaux de cuisine), de trocs de cultigènes ou de produits forestiers (gibier, fruits), ceci contre des marchandises et, surtout, des aliments industriels (riz, farine de manioc, sel, sucre, conserves) .

Cet équilibre ne pouvait être que précaire, sans cesse menacé par l'augmentation du nombre des orpailleurs, la propagation des maladies (paludisme, grippe, etc.) et la croissance des conflits à propos d'échanges. Elle ne put se maintenir que parce que la présence massive des chercheurs d'or n'a duré qu'un an et demi (mi-88 à janvier 90). A plus long terme, la densification rapide des placers le long du haut Mucajaí aurait rendu impossible toutes activités productives traditionnelles et les maladies des chercheurs d'or auraient fini par décimer les quelques centaines de Yanomami de Homoxi: la mortalité moyenne des communautés affectées par le garimpo durant les années 1988-89 a été estimée de 15 à 60 \% (APC 1990).

Finalement, c'est après le retrait des orpailleurs par la PF, à partir de janvier 1990, au plus fort de l'épidémie de paludisme qui ravagea la région, que les Tirei quitteront leur site du Narahipi $u$, où ils s'étaient maintenus jusque là. Toutefois, ils ne s'éloigneront pas pour autant de la zone la plus dégradée des placers le long du haut Mucajaí, à l'instar des groupes de Xere $u$ et Yaritha. Ils tenteront ainsi de maintenir leur système de relations avec les Blancs les équipes d'assistance (PF, FUNAI, FNS) ayant remplacé les orpailleurs - plutôt que d'optimiser à nouveau leur système productif dans sa configuration traditionnelle en s'éloignant de la zone d'orpaillage. Alors que le décès d'un grand nombre d'ainés avait désorganisé les activités collectives et que les maladies immobilisaient constamment le reste de leur population active, les Tirei renoncèrent à reconstruire leur communauté loin des Blancs.

\section{Les Blancs changent, le système de dépendance demeure}

Réfugiés dans des campements de bâches de plastique venant des camps d'orpailleurs, les Tirei entameront, après l'évacuation des garimpeiros, ${ }^{34}$ une décennie de déplacements successifs dans le sillage et la dépendance des équipes venues leur porter assistance. Au début 1990, ils quitteront définitivement le Narahipi $u$, pour s'approcher de leur poste provisoire de la piste Baiano formiga (bas Xere $u$ et Napëroi u). Puis, après leur départ vers la piste Jeremias, devenue Poste Homoxi, les Tirei reprendront leur migration en plusieurs étapes. Ils se sépareront des Xere $u$, vers le Apiahipi $u$, puis s’établiront dans le voisinage immédiat de la piste de Homoxi, pour, finalement, se regrouper, en 2001, en une seule maison collective, à moins de cent mètres du poste lui-même.

Les Tirei désignent les années 90 comme le «temps de la Fondation» (hutasao tëhë), en référence à la FNS, acteur principal de la structure d'assistance du Poste Homoxi. C’est en

\footnotetext{
${ }^{34}$ Des groupes d’orpailleurs reviendront toutefois dans la région, mais à une échelle bien moindre, au moins jusqu’en 1998. Les Yanomami rapportent que ces retours bénéficièrent parfois de la complicité des employés de la FUNAI ou de la FNS, qui leur vendaient du ravitaillement et/ou leur rendaient les équipements saisis par la Police fédérale. La seule période véritablement libre de chercheur d’or se situerait donc entre 1999 et 2002, date de notre étude.
} 
effet, au personnel de cette administration sanitaire que s'adresseront, durant cette période, non seulement les requêtes de soins mais encore - et surtout - les demandes constantes de soutien alimentaire et de biens manufacturés (outils, hamacs, vêtements), transformant le poste de santé, du point de vue yanomami, en substitut d'un campement d'orpailleurs. Ce malentendu engagera peu à peu le personnel sanitaire dans un système de relations économiques sans rapport avec ses fonctions et d'autant moins tenable que la FNS maintenait le plus souvent ses agents dans l'abandon le plus complet. Durant presque une décennie ce rôle dévolu, malgré elles, aux équipes de terrain de la FNS - aggravé par l'irritation des Yanomami devant leur inefficacité à contrôler la surmortalité due à l'épidémie de paludisme donna lieu à des conflits incessants, émaillés de pillages du poste et de menaces de violences. Le 5 août 1999 le journal du poste Homoxi (FNS) notait ainsi encore: "Les indiens de ce poste sont armés et menacent quelquefois notre équipe avec des machettes et des fusils après que nous ayons, à plusieurs reprises, refusé de leur donner des hamacs et de la nourriture ».

A partir de l'année 2000, les moyens investis par les ONG auxquelles a été transférée la responsabilité de Homoxi (URIHI pour la santé, CCPY pour l'éducation et l'environnement) ont produit une considérable amélioration de la situation sanitaire de la région. Elles ont aussi permis la mise en place d'un système de relations économiques et sociales qui ouvre la voie à une sortie du modèle de dépendance hérité du «temps des orpailleurs », que les Tirei ont tendance à reproduire avec tout établissement blanc - quel qu'il soit - implanté sur son territoire.

\section{Impacts économiques et territoriaux du garimpo et stratégies d'adaptation des Tirei}

\section{Diminution des ressources agricoles}

Le premier impact des activités d'orpaillage sur l'espace productif des Tirei a été la destruction de de leurs jardins sur le bas Narahipi u par l'ouverture de la piste Macarrão et par la multiplication des sentiers ouverts entre les placers le long du haut Mucajaí. A ces dégradations vinrent s'ajouter une pression croissante sur les ressources agricoles restantes en fonction des demandes de troc des orpailleurs (ou des vols), puis la désorganisation des activités agricoles en raison de l'aggravation de la situation sanitaire (paludisme, infections respiratoires). Cette accumulation de facteurs adverses eut deux conséquences:

- l'abandon des maisons collectives de la zone riveraine, soumise à une exploitation intense dans le vacarme incessant des motopompes et des atterrissages d'avions et d'hélicoptères, et le repli sur celles qui se trouvaient en amont des tributaires de la rive gauche du haut Mucajaí;

- une réduction de la taille des nouveaux jardins et une dépendance alimentaire croissante envers les orpailleurs, via une routine de demande d'aliments ou de recherche de rétribution pour de menus services (la main d'œuvre yanomami étant considérée inadaptée à l’orpaillage mécanisé).

La superficie réduite des jardins des Tirei en relation à leur population (environ 4,5 ha de jardins ouverts en 2002), leur tendance à l'instabilité résidentielle (visites fréquentes chez leurs alliés ou en forêt) et leur propension durable à la dépendance alimentaire vis-à-vis du Poste Homoxi, révèlent leur difficulté à s'émanciper du modèle de survie adopté durant l'époque du garimpo (1988-89).

Le journal du poste de santé (FNS) les décrivait ainsi (29/1/1994) comme « un groupe qui a pratiquement cessé toute activité agricole et dépend, pour sa nourriture, de ses demandes à la FUNAI et à la FNS ». En 1999, un médecin (Dr P.Basta) notait également dans un rapport sur Homoxi « (..) la demande constante de nourriture de la part des Yanomami » et ajoutait qu'elle n'était plus liée à un problème de malnutrition mais à «de nouvelles habitudes 
alimentaires" dues "aux distributions d'aliments par les orpailleurs, qui utilisaient ce stratagème pour conquérir la sympathie des indiens et rétribuer leurs services dans les garimpos ».

\section{Restriction des ressources de la pêche et réaménagement de la chasse}

L’orpaillage a profondément et durablement affecté le cours supérieur du Mucajaí et sa faune aquatique. Les Tirei relatent que le lit de cette rivière était plus large autrefois et qu'elle inondait la forêt loin de ses berges durant la saison des pluies (pic en juillet), temps durant lequel les poissons migraient dans la forêt inondée à la recherche d'une alimentation abondante. Aujourd'hui, le cours du fleuve est étroit et peu sinueux, encaissé entre des monticules de graviers extraits des placers. Ses berges ont été défrichées puis dévastées par les motopompes. Elles sont bordées d'innombrables lagunes aux eaux troubles, dont la profondeur varie entre deux et quatre mètres, vestiges des activités d'orpaillage, que les Yanomami nomment les « trous de l'or » (oru pëkapë).

L'invasion rapide du bassin du haut Mucajaí par les orpailleurs a également eu un impact très négatif sur la productivité de la chasse yanomami. Les Tirei ont du cesser toute activité cynégétique le long du Narahipi $u$ et des autres petites rivières voisines, où se déployaient leurs itinéraires de chasse quotidienne, du fait des nuisances liées aux activités d'orpaillage. La densité de l'occupation humaine et le vacarme constant des pompes et des mouvements aériens ont peu à peu mis en fuite la plupart du gibier de la zone riveraine. Par ailleurs, les orpailleurs pratiquaient une chasse intensive, au fusil ou avec des pièges, de jour comme de nuit, surexploitant rapidement le gibier, même dans les zones plus lointaines ${ }^{35}$. Ils ont, enfin, introduit divers animaux domestiques (chiens, chats, canards, poules et porcs) dont les maladies infectieuses ont pu avoir une influence néfaste sur la faune locale ${ }^{36}$.

Dès lors, les Yanomami du haut Mucajaí ont commencé à distinguer deux types d'espaces, opposant régions d'orpaillage (espaces riverains) et zones intactes (hautes terres) : urihi wãriopëha, "zones de forêt détruite » et urihi totihiopëha, "zones de belle forêt» (ou urihi temiopëha, « zones de forêt saine »). Afin de compenser l'absence de gibier dans la zone des placers, les Tirei ont commencé à intensifier l'usage (habituellement sporadique) de leurs circuits de chasse et collecte à longue distance ${ }^{37}$, contournant les espaces de «forêt détruite " afin de pouvoir accéder aux lointains espaces de « forêt saine » encore giboyeux.

Cette stratégie d'adaptation de la chasse aux contraintes spatiales et écologiques de l'orpaillage a tenu sur une période courte (environ un an et demi, entre 1988 et 1990). Elle semble avoir constitué pour les Tirei un compromis, malaisé mais viable, dans la mesure où ils avaient choisi de rester sur place pour maintenir leurs échanges avec les orpailleurs. Ainsi, le choix stratégique du groupe a-t-il été, avant la catastrophe sanitaire du début des années 90 , de faire appel à une dimension traditionnelle de l'économie yanomami - le système des expéditions collectives à longue distance - en l'adaptant au contexte particulièrement atypique et contraignant de l'invasion des garimpos. On sait, ainsi, que les groupes yanomami isolés passaient jusqu'à 40 \% de l'année en expédition de chasse et de collecte en forêt (Good 1989: 123-128, 165).

\footnotetext{
${ }^{35}$ Ils ont toutefois négligé plusieurs espèces importantes pour les Yanomami (comme les singes) qu’ils ont simplement mises en fuite. Inversement, certaines autres espèces très visées ont rapidement diminué (pécaris à collier, daguets, tapirs, etc.) ou même disparu dans la région (caïmans, tamanoirs, tatous).

${ }^{36}$ Une étude sur la disparition des hordes de pécaris à lèvre blanche dans le territoire yanomami a invoqué la possibilité d’une contamination par les porcs domestiques (Fragoso 1997). Il s'agit d'une perte considérable : Sponsel (1981: 187), a montré qu’ils représentaient 74\% du poids total du gibier chassé (Yanomami septentrionaux, 1974-75).

${ }^{37}$ Ces itinéraires, ponctués par des campements, sont habituellement utilisés lors des chasses de longue durée destinées à fournir le gibier boucané des cérémonies funéraires d'alliance ou lors de longs séjours de chasse et collecte en forêt, notamment durant la maturation des nouveaux jardin.
} 


\section{Une dépendance stratégique}

La vision des Tirei comme un groupe anomique, leitmotiv des rapports de santé des années 90, néglige les paramètres socioéconomiques de la situation de ce groupe après l'expulsion soudaine des chercheurs d'or (janvier 1990) et la nécessité dans laquelle il se trouvèrent de reproduire - cette fois en relation avec la structure d'assistance locale l'équilibre précaire du modèle d'adaptation élaboré durant le « temps des garimpeiros » et qui venait d'être brutalement remis en question.

Du fait de l'impact territorial, environnemental et social de la présence des garimpeiros sur leur système productif (agriculture, pêche, collecte et chasse), les ressources acquises auprès des Blancs étaient en effet devenues un élément fondamental de l'économie du groupe, tant pour renforcer son apport alimentaire que pour maintenir ses relations de troc et d'alliance avec les groupes voisins (Xere $u$ et Wiramapi u/Yaritha), réfugiés dans une zone épargnée par l'orpaillage, et dont la solidarité alimentaire et politique a souvent du être mise à contribution au cours des années 1990. La dégradation de l'environnement des Tirei constatée par notre étude et le cycle guerrier qui les opposait à leurs voisins du nord (Kurema $u$ et Huruna) laissent penser qu'il s'agissait là d'une stratégie plus que réaliste au vu de leur situation et de leurs possibilités d'action. Ainsi, si les contraintes affectant la chasse ont pu être en partie compensées par une redéfinition spatiale de leur usage des ressources, celles, plus drastiques encore, qui entravaient les activités agricoles, l'ont été par une « dépendance stratégique » des garimpos en matière de ressources alimentaires (riz, farine de manioc, haricots rouges, sucre, sel, conserves) et de biens manufacturés (outils, marmites, vêtements, hamacs, fusils et cartouches) ; ces derniers servant également à négocier de longs séjours chez leurs alliés en cas de disette ou de menace guerrière.

Dans ce contexte, sans le savoir, le personnel du Poste Homoxi ne put échapper totalement au rôle que les Tirei lui assignèrent dans la réactualisation du système de dépendance qui leur était familier depuis l'époque du garimpo. Cette situation a bien été notée en 1991, par un autre médecin (Dr M. Pellegrini), qui observa que le personnel du poste s'efforçait, pour faciliter son travail et apaiser une situation de conflit chronique avec les Indiens, de privilégier le groupe de Tirei en relation aux autres communautés, à la fois en rétribuant leurs services avec des aliments «de meilleure qualité et en plus grande quantité que ceux fournis aux malades » et en les incitant à venir se faire soigner au poste en leur promettant des rations alimentaires.

L'ensemble de ces faits doit, ainsi, nous inciter à réévaluer l'image négative dont pâtissent généralement les groupes yanomami associés aux garimpos en révélant dans leurs choix des stratégies propres et souvent efficaces, à même de garantir leur survie dans un contexte environnemental et économique critique dont il n'était plus temps de s'échapper une fois refermé sur eux le piège de l'invasion massive des garimpeiros. On peut, ainsi, mieux comprendre la justification que donnent les Tirei de leur choix surprenant de demeurer sur le bas Narahipi $u$, dans la zone centrale des placers, durant l'époque du garimpo.

D’après les témoignages recueillis, le groupe a été rapidement encerclé sur ce site par plusieurs milliers d'orpailleurs, le haut Mucajaí et tous ses affluents se trouvant rapidement pollués par l'exploitation de leurs alluvions aurifères ${ }^{38}$. Le Narahipi $u$, que les Tirei ont, selon leur terme, " protégé » contre les chercheurs d'or, est ainsi devenu pour eux un " sanctuaire », zone de résidence et unique rivière " propre » de la région où il soit encore possible de puiser de l'eau potable, de pêcher et de collecter divers amphibiens et crustacés. C'est donc à partir de ce «sanctuaire » résidentiel, une fois garanties des ressources hydriques et aquatiques

\footnotetext{
${ }^{38}$ Au point que les orpailleurs devaient eux-mêmes creuser des puits pour leur approvisionnement en eau potable.
} 
fondamentales, que les réaménagements territoriaux et économiques subséquents du groupe ont pu être menés à bien.

\section{Conclusion}

La ruée vers l'or du Roraima a, à la fin des années 1980, brutalement confronté des groupes Yanomami encore isolés avec une dynamique économique et sociale exogène des plus agressives. Un épisode de la conquête intérieure qui a marqué cinq siècles d'histoire du Brésil a été ainsi réédité, avec son lot de violences et, surtout, de décimations épidémiologiques. En cette fin du XXème siècle de nouveaux facteurs sont toutefois intervenus pour que l'histoire ne se répète pas à l'identique.

Sur le plan global, la préoccupation croissante pour la protection de l'environnement et les droits des minorités, au Brésil et dans le monde, a permis de contenir l'invasion du territoire yanomami par les orpailleurs, évitant qu'elle ne se transforme en fait accompli. De fait, après plusieurs opérations de la $\mathrm{PF}$ et, malgré quelques enclaves résiduelles, les garimpeiros ont été expulsés de la "Terre Indigène Yanomami » tandis qu'une meilleure assistance sanitaire a permis à cette ethnie de retouver son essor démographique et culturel. Il s'agit là d'une situation nouvelle dans l'histoire des ethnies amazoniennes car, malgré leur tragique expérience des années 70 (route Perimetral Norte) et 80 (ruée vers l'or), les Yanomami jouissent aujourd'hui d'un certain répit qui leur permet d'acquérir de nouveaux savoirs et d'élaborer de nouvelles stratégies de relations avec les Blancs.

Sur le plan local, la situation observée à Homoxi a démontré la flexibilité du système économique et social yanomami et sa capacité à générer des adaptations rapides aux situations les plus adverses. Ainsi, malgré un rapport de force très défavorable, les Tirei ont-ils réussi à se maintenir en tant que groupe et à conserver le contrôle de leur territoire. S'adaptant à l'invasion des orpailleurs puis à la présence de la structure d'assistance qui les a remplacés, ils ont aujourd'hui acquis dans la région une position dominante en terme d'accès aux soins, à l'éducation et aux biens manufacturés. Pour parvenir à ce résultat, ils ont développé un nouvel usage de leur espace basé sur un système de résidences multiples et sur une adaptation de traits fondamentaux de leur suystème productif, tels que la réorganisation de leurs parcours de chasse ou le rythme des migrations temporaires. Savoir dans quelle mesure cette adaptation est circonstancielle ou s'il s'agit d'un changement de paradigme partagé par d'autres groupes yanomami fait l'objet d'une nouvelle recherche commencée par notre équipe à partir des résultats présentés dans cet article. 


\section{Bibliographie}

ALBERT, B. [1987], « Les Indiens et la Nouvelle République », Les Temps Modernes 491 : 121-141.

. [1990], «Développement Amazonien et Sécurité Nationale: les Indiens Yanomami face au projet 'Calha Norte' », Ethnies 11-12: 116-127.

[1992], «Indian lands, environmental policy, and military geopolitics in the development of the Brazilian Amazon: The case of the Yanomami », Development and Change 23 (1): 35-70.

- [1994], «Indiens Yanomami et chercheurs d'or au Brésil. Le massacre de Haximu », Journal de la Société des Américanistes 80 : 250-57.

ALBERT, B. et GOMEZ, G. [1997], Saúde Yanomami. Um manual etno-lingüístico. Belém : Museu Goeldi, 304 p.

ALBERT, B. et KOPENAWA, D. [2003], Yanomami. L'esprit de la forêt. Paris : Actes SudFondation Cartier pour l'Art Contemporain, $206 \mathrm{p}$.

ALBERT, B. et LE TOURNEAU, F.-M. [2004], «Florestas Nacionais na Terra Indígena Yanomami : um cavalo de Tróia ambiental ? », in: Terras indígenas e unidades de conservação da natureza. O desafio das sobreposições. São Paulo: Instituto Socioambiental, pp. 372-383.

APC (Ação pela Cidadania) [1990], Yanomami: a todos povos da terra. São Paulo: CCPY/Cedi/Cimi/NDI, 48 p.

CAPOBIANCO, J.P. (éd.) [2001], Biodiversidade na Amazônia Brasileira. São Paulo: Editora Estação Liberdade/Instituto Socioambiental, 540 p.

CCPY, [1989], "Mineração: o esbulho das terras Yanomami. Histórico das invasões 19751989”, Urihi 11: 10-11.

CLEARY, D. [1990], Anatomy of the Amazon Gold Rush. London: MacMillan, 287 p.

FRAGOSO, J. [1997], « Desapariciones locales del baquiro labiado (Tayassu pecari) en la Amazonia, migracion, sobre-cosecha, o epidemia ? », in: T.G. Fang et al. (éds.): Manejo de fauna silvestre en la Amazônia. Lima: UNAP, Université de Floride, UNDP-GEF, Universidad Mayor de San Andrés, pp. 309-312.

GEFFRAY, C. [1995], Chroniques de la servitude en Amazonie brésilienne. Paris : Karthala, $185 \mathrm{p}$.

GOOD, K. [1989], Yanomami Hunting Patterns: Trekking and garden relocation as an adaptation to game availability in Amazonia, Venezuela. Thèse de Doctorat, Université de Floride, 177 p.

LE TOURNEAU, F.M. [1999], Étude des paysages du littoral amazonien à partir d'images de radar à synthèse d'ouverture. Thèse de Doctorat, Université de Marne-la-Vallée, 300 p.

MACMILLAN, G. [1995], At the End of the Rainbow ? Gold, Land and People in the Brazilian Amazon. Londres: Earthscan, 199 p.

PITHAN, O.A., CONFALONIERI, U.E.C. et MORGADO, A.F. [1991], “A situação de saúde dos índios Yanomami: diagnóstico a partir da casa do índio de Boa Vista, Roraima, 19871989”, Cadernos de Saúde Pública 7(4): 563-580.

RAMOS, A.R. [1998], Indigenism: Ethnic Politics in Brazil. Madison, Wisconsin: The University of Wisconsin Press, 326 p.

RAMOS, A.R., M. LAZARIN et G. GOMEZ [1987], Yanomami em tempo de ouro: relatório de Pesquisa, in: Culturas indígenas de la Amazônia, L.Yáñez-Barnuevo (éd.). Madrid: Biblioteca Quinto Centenário, pp.73-84.

RICARDO, F. (éd.) [1999], Interesses minerários em terras indígenas na Amazônia Legal Brasileira. Documentos do ISA 6. São Paulo: Instituto Socioambiental, 101 p. 
SPONSEL, L. [1981], The hunter and the hunted in the Amazon: an integrated biological and cultural approach to the behavioral ecology of human predation. Thèse de Doctorat, Université de Cornell, 495 p.

TAYLOR, K.I. et RAMOS, A.R. (éds.) [1979], The Yanoama in Brazil 1979. IWGIA Document 37. Copenhague: IWGIA, $170 \mathrm{p}$.

WILBERT, J. et SIMONEAU, K. (éds.) [1990], Folk Literature of the Yanomami Indians. Los Angeles: UCLA Latin American Center Publications, 789 p. 